\title{
METHOD OF CHARACTERIZATION OF COMPLEX AND DEGENERATED EPITAXIAL STRUCTURES FROM ANALYSIS OF MAGNETORESISTANCE OSCILLATIONS
}

\author{
R. L. AULOMBARD, C. BOUSQUET, C. BERNARD-MERLET, A. RAYMOND \\ and J. L. ROBERT
}

Centre d'Etudes d'Electronique des Solides $\left(^{*}\right)$,

Université des Sciences et Techniques du Languedoc, place Eugène-Bataillon, 34060 Montpellier Cedex, France

\section{P. RUB and C. PICOCHE}

S.N.C.I., C.N.R.S., avenue des Martyrs, 38000 Grenoble, France

\begin{abstract}
Résumé. - Nous montrons qu'à partir du traitement par transformée de Fourier discrète du signal de magnétorésistance obtenu à basse température, nous pouvons déterminer rapidement et précisément la concentration en porteurs de charge de toute structure complexe dégénérée pouvant être utilisée comme dispositif. Nous traitons ici le cas de multicouches $n^{+} n^{++}$d'arséniure de gallium utilisées dans les dispositifs hyperfréquences. Nous montrons également qu'à partir de mesures effectuées sous pression hydrostatique, cette méthode d'analyse peut être comparée à celle déjà proposée pour des structures simples $\left({ }^{1}\right)$.
\end{abstract}

\begin{abstract}
Using a discrete Fourier transform on the magnetoresistance signal, obtained at low temperature we show that it is possible to determine easily and accuratly the carrier concentration of all complex and degenerated semiconductors. We give the example of gallium arsenide $n^{+} n^{++}$ multilayers used in microwave devices. From measurements under hydrostatic pressure we show also that this method of characterization is consistent with the one previously given for simple structures $\left({ }^{1}\right)$.
\end{abstract}

1. Introduction. - In a previous paper we have proposed a method of characterization [1] of degenerated semiconductors, which allows for the determination of both compensation and spatial fluctuations of impurities (afterwards called method I). As a result the method can be used in the study of correlation between the growing process and the quality of the crystal. It can be only applied in the case of bulk samples or single epitaxial layer.

Now we propose a new method (method II) for characterization of all the complex epitaxial structures and it is applied here in the particular case of gallium arsenide $n^{+} n^{++}$multilayers as used in microwave devices. This direct method is based on the Fourier analysis of the magnetoresistance oscillations (Shubnikov-de Haas effect) obtained at low temperature. This method is of a great interest because it allows for the characterization of each layer without knowing the dimensions of the structures. Particularly it gives directly the values of the carrier concentrations in the layers. Furthermore we show that the amplitude of fluctuations of impurities can be deduced by analysis

(*) Associé au C.N.R.S., L.A. 21.

( $\left.{ }^{1}\right)$ Raymond, A., Robert, J. L. and Pistoulet, B. (1977), Gallium arsenide and related compounds (Edinburgh 1976) Int. Conf. Ser. 33a (1976) 105. of Shubnikov-de Haas effect under hydrostatic pressure. The main advantage of this method compared to the previous one lies in the fact that now it is not necessary to measure separatly the mobility of the carriers, that is difficult in many cases.

In order to verify this model we have applied this second method to the case of a single epitaxial layer of gallium arsenide. We show that the results are consistent with those obtained by the first one.

2. Amplitude of magnetoresistance oscillations and relaxation times in presence of potential fluctuations (method I). - The expression of the amplitude of the magnetoresistance oscillations in the case of ideal crystal is given by :

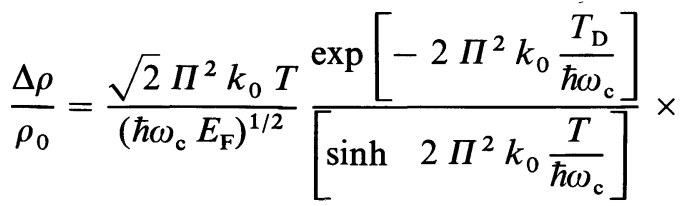

$$
\begin{aligned}
& \times \sin \left(\frac{2 \Pi F}{B}+\gamma\right)
\end{aligned}
$$

where $F=$ frequency of the oscillations

$$
=\frac{\hbar}{2 e}\left(3 \Pi^{2} n_{0}\right)^{2 / 3} \text {. }
$$


$T_{\mathrm{D}}=$ Dingle temperature $=\frac{\hbar}{2 \Pi k_{0}} \frac{1}{\tau}$.

$\tau=$ cyclotron relaxation time.

$E_{\mathrm{F}}=$ Fermi level $=\left(3 \Pi^{2}\right)^{2 / 3} \frac{\hbar^{2}}{2 m_{\mathrm{d}}^{*}} n_{0}^{2 / 3}$.

$\omega_{\mathrm{c}}=$ cyclotron frequency $=\frac{e B}{m^{*}}$.

$\gamma=$ phase constant.

If we take into account a medium range disorder in the spatial distribution of impurities inside the crystal [2] the main parameters used in the analysis of Shubnikov-de Haas effect are strongly affected.

In this case of a real crystal we have shown that the expression [1] must be replaced by :

$$
\begin{array}{r}
\frac{\Delta \rho}{\rho_{0}}=\int_{0}^{\infty} \frac{\Pi^{2} \sqrt{2} k_{0} T}{\hbar e\left(\frac{B F}{m^{*} m_{\mathrm{d}}^{*}}\right)^{1 / 2}} \frac{\exp \left[-\frac{\Pi m^{*}}{e B \tau}\right]}{\sinh \left[\frac{2 \Pi^{2} k_{0} m^{*} T}{\hbar e B}\right]^{2}} \times \\
\times \sin \left(\frac{2 \Pi F}{B}+\gamma\right) P(F) \mathrm{d} F .
\end{array}
$$

Assuming a lorentzian distribution of the carrier density i.e. of the frequencies :

$$
P(F)=\frac{2}{\Pi \Delta F} \frac{1}{1+\left[\frac{2\left(F-F_{0}\right)}{\Delta F}\right]^{2}}
$$

where $F_{0}$ is the frequency corresponding to the average carrier concentration $n_{0}$.

We have obtained a new expression of the amplitude $A$ of the magnetoresistance oscillations :

$$
\begin{array}{r}
A=\frac{\Pi^{2} \sqrt{2} k_{0} T}{\hbar e\left(\frac{B F}{m^{*} m_{\mathrm{d}}^{*}}\right)^{1 / 2}} \frac{\exp -\frac{\Pi m^{*}}{e B \tau}}{\sinh \left[\frac{2 \Pi^{2} k_{0} m^{*} T}{\hbar e B}\right]} \times \\
\times \exp \left(-\frac{\Pi \Delta F}{B}\right) .
\end{array}
$$

The expressions of both cyclotron and conductivity relaxation times, including spatial fluctuations of impurity and compensation are given ref. [1] :

$$
\begin{aligned}
& \frac{1}{\tau}=\frac{1}{\tau\left(n_{0}\right)}\left(1+k_{1}^{\prime} \frac{\Delta n}{n_{0}}+2 k_{2}^{\prime} \frac{N_{A_{0}}}{n_{0}}\right) \\
& \frac{1}{\tau_{\sigma}}=\frac{1}{\tau_{\sigma}\left(n_{0}\right)}\left(1+k_{1} \frac{\Delta n}{n_{0}}+2 k_{2} \frac{N_{A_{0}}}{n_{0}}\right)
\end{aligned}
$$

where $\tau\left(n_{0}\right)$ and $\tau_{\sigma}\left(n_{0}\right)$ are the expressions of the cyclotron and conductivity relaxation times for the case of impurity scattering (1) (3) and including quantum corrections. From the analysis of Shubnikov-de Haas effect and mobility measurements both the spatial fluctuations of carrier concentrations $\Delta n / n_{0}$ and compensation $N_{A_{0}} / n_{0}$ can be deduced easily in the case of single layer and bulk samples. However it is clear that this method cannot be used directly in the case of multilayers.

3. Method of characterization of complex structures (method II). - For complex structures a direct method, using a Fourier transform, leads to the determination of the carrier densities. The width of the frequency spectrum results of the effect of temperature and of the broadening of the Landau levels due to scattering and disorder.

3.1 Data aCQuisition AND PROCESSING. - The magnetoresistance voltage is measured directly by an integrater digital voltmeter monitored by a scanner on line with a computer (model PDP 11/20). The acquisition is made on the $100 \mathrm{mV}$ range and $200 \mathrm{~ms}$ integrating time; so the resolution is equal to $1 \mu \mathrm{V}$. Two or five minutes sweeping rate of the magnetic field can be choosen and the curves are defined by 400 or 1000 points. Then the recorded informations are transfered from the previous computer to another (model PDP 10) on which all the calculations are made.

For the method I, the peaks of magnetoresistance are localized; the envelopes are drawn and the mean value of the magnetoresistance is withdrawn to the direct signal.

For the direct analysis in which a Fourier transform is used (method II) the same process is made in order to suppress the d.c. component in the case of single layer. For more complex structures a polynomial approximation method is used. Then a transform Fourier program is applied (4). This Fourier transform is modified in such a manner that it is amenable to digital computation. This modified Fourier transform, called discrete Fourier transform (DFT) is to approximate as closely as possible the continuous Fourier transform. Then the power spectrum is obtained and using an inverse discrete Fourier transform (IDFT) we obtain the initial signals for each frequency.

\subsection{EXPERIMENTAL ReSUlTS. - i) Determination of} the carrier density. - Typical results of Shubnikovde Haas effect that we have obtained on single layer and multilayers of gallium arsenide are given figure 1 and 2 .

The corresponding power spectra are given figure 3 and 4.

The values of the mean carrier densities $n_{0}$ are deduced from the frequencies $F_{0}(2)$. An epitaxial single layer $n^{++}$and epitaxial multilayers $n^{+} n^{++}$ have been prepared simultaneously in the same apparatus and as expected we observe that the $n^{++}$ layers have the same doping. It is clear that such a result cannot be obtained so easily and so accuratly by other methods. 


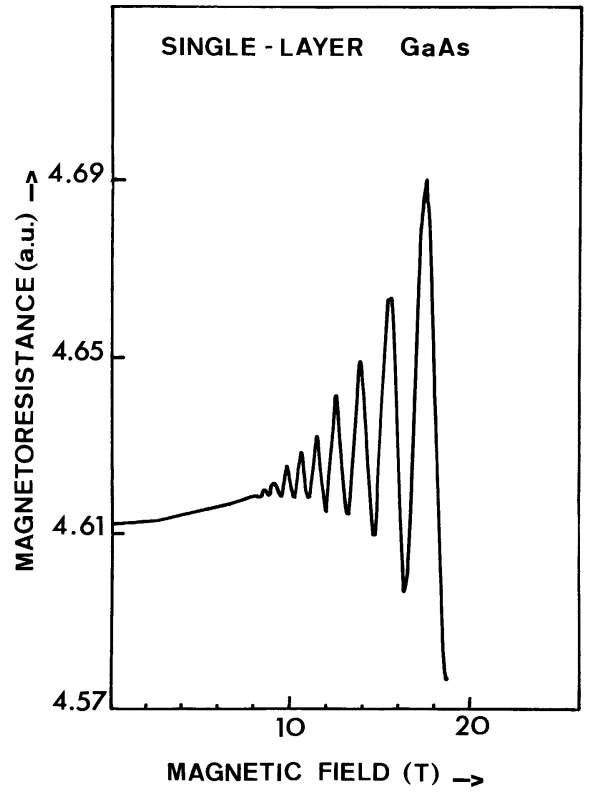

FIG. 1. - Longitudinal magnetoresistance for a single layer of gallium arsenide.

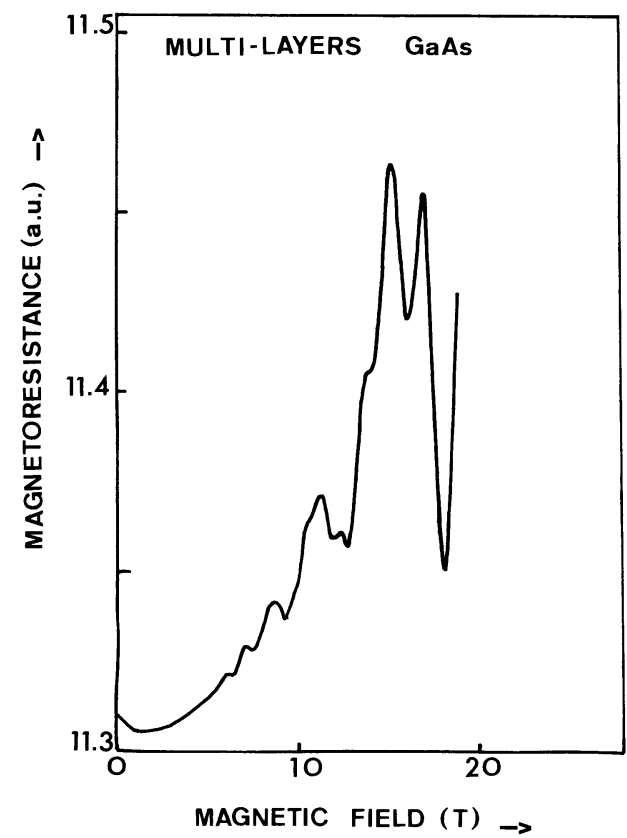

FIG. 2. - Longitudinal magnetoresistance for $n^{+} / n^{++}$multilayers of gallium arsenide.

The numerical values obtained from the frequency spectra of figure 3 and figure 4 are :

Fig. $3 \quad F_{0}=132.8 \mathrm{~T} \quad n_{0}^{++}=8.65 \times 10^{24} \mathrm{~m}^{-3}$

Fig. $4 \quad F_{0}^{\prime}=37.5 \mathrm{~T} \quad n_{0}^{\prime+}=1.30 \times 10^{24} \mathrm{~m}^{-3}$ $F_{0}^{\prime \prime}=132 \mathrm{~T} \quad n_{0}^{\prime \prime+}=8.58 \times 10^{24} \mathrm{~m}^{-3}$.

ii) Determination of fluctuations of impurities. - In order to determine the amplitude of fluctuations of impurities, we compare the half width $\Delta F_{\mathrm{T}}$ of the fre-

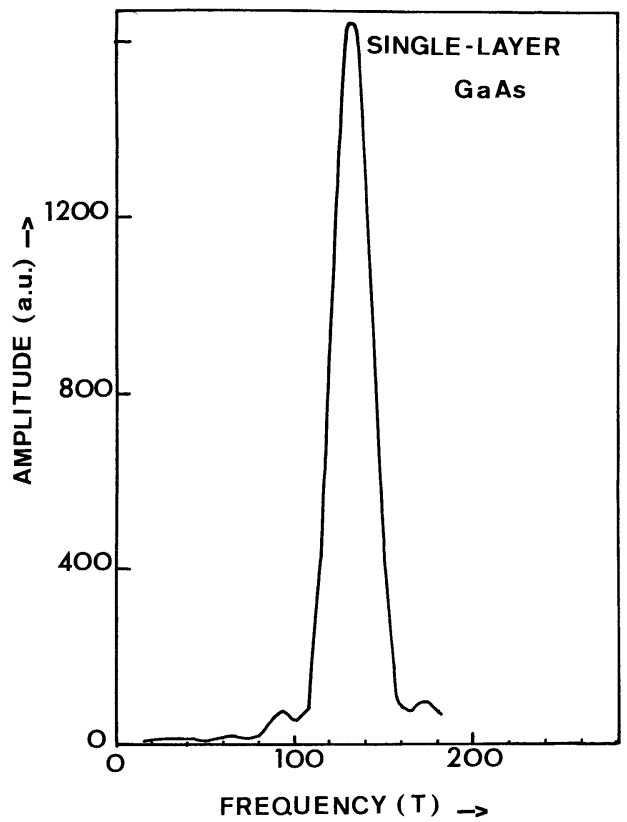

FIG. 3. - Frequency analysis of the longitudinal magnetoresistance oscillations described on figure 1.

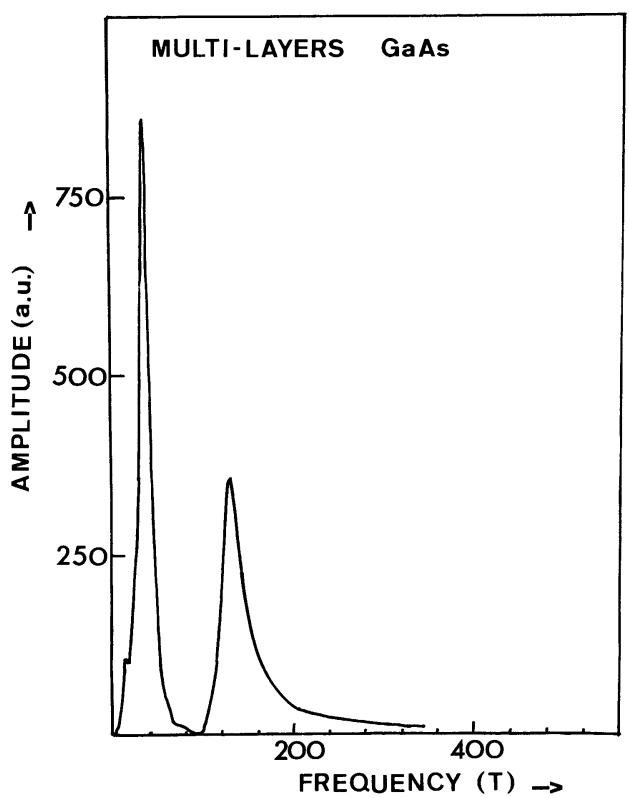

FIG. 4. - Frequency analysis of the longitudinal magnetoresistance oscillations described on figure 2 .

quency spectrum without and under hydrostatic pressure.

Indeed $n_{0}$ and $\Delta n / n_{0}$ do not vary with the pressure and the part of $\Delta F_{\mathrm{T}}$ due to the disorder (called $\Delta F_{\mathrm{i}}$ ) does not depend on the effective mass $m^{*}$. This result can be seen, if we consider the theoretical expression (4)

$\exp \left[-\frac{\Pi \Delta F_{\mathrm{i}}}{B}\right]=\exp \left[\frac{-\Pi^{2} \hbar}{e B}\left(\frac{\Pi}{3}\right)^{1 / 3} n_{0}^{2 / 3} \frac{\Delta n}{n_{0}}\right]$.

On the other hand, the part of $\Delta F_{\mathrm{T}}$ due to the effect of 
scattering and temperature is a function of the effective mass.

Using the expression of the cyclotron relaxation time $\tau$ given for impurity scattering (1); it can be shown easily that $1 / \tau$ does not depend in first approximation of $m^{*}$ : then the quantity $\Pi m^{*} / e B \tau$ (eq. (4)) is proportional to the effective mass.

The effective mass $m^{*}$ increases with increasing hydrostatic pressure : this gives an increase of the part of $\Delta F_{\mathrm{T}}$ due to the effect of collision and temperature. This increase is proportional to the effective mass one.

In order to assert this result, we have performed experiments of Shubnikov-de Haas effect on a single layer of gallium arsenide under hydrostatic pressure up to $7.5 \mathrm{kbar}$. The increase of the effective mass is deduced from the increase of the resistivity with pressure. The resistivity varies indeed with pressure like the square of the effective mass (3). From the value of $\Delta F_{\mathrm{i}}$ due to disorder we obtain the value of $\Delta n / n_{0}$ :

$$
\frac{\Delta F_{\mathrm{i}}}{F_{0}}=\frac{2}{3} \frac{\Delta n}{n_{0}} .
$$

All numerical values are given in the following table. The analysis of the Shubnikov-de Haas effect by the method I gives results in good agreement with those obtained by method II.

$$
\begin{aligned}
& \text { Method I }
\end{aligned}
$$

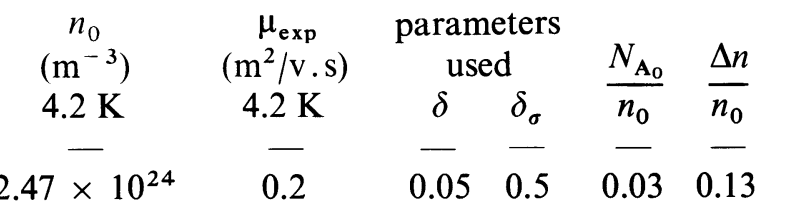

4. Conclusion. - In short we have developed a method which allows us from a Fourier analysis, the determination of the carrier density of all complex degenerated structures.

\section{Method II}

$$
\begin{aligned}
& \begin{array}{cccc}
F_{0} & n_{0} & \frac{\Delta F_{7.5 \mathrm{kbar}}-\Delta F_{0 \mathrm{kbar}}}{F_{0}} & \frac{\rho_{7.5 \mathrm{kbar}}}{\rho_{0 \mathrm{kbar}}}
\end{array} \\
& \text { - } \quad 249 \times 10^{24} \quad \overline{013} \quad \overline{117}=0 .
\end{aligned}
$$

Furthermore, it is also possible to have information on the microscopic quality of the crystal.

Acknowledgments. - The authors would like to thank Dr. L. Hollan (L.E.P., 95, Limeil Brevannes, France) for supplying the GaAs samples and Dr. A. Eberhard (C.N.R.S., Grenoble, France) for his helpfull discussions and the Fourier transform program.

\section{References}

[1] Raymond, A., Robert, J. L. and Pistoulet, B., Gallium arsenide and related compounds (Edinburg 1976) Int. Phys. Conf. Ser. 33a (1977) 105.

[2] Robert, J. L., Pistoulet, B., Raymond, A., Aulombard, R. L., Bernard, C. and Bousquet, C., Revue Phys. Appl. 13 (1978) 246.
[3] Zawadski, W. and Szymanska, W., J. Phys. Chem. Solids 32 (1971) 1151

Zawadski, W. and Szymanska, W., Phys. Status Solidi (b) 45 (1971) 415

[4] Eberhard, A., Thesis, Grenoble, June 1970. 